\title{
O Enfermeiro na Estratégia Saúde da Família no enfrentamento da COVID-19: Revisão Integrativa
}

\author{
The Nurse in the Family Health Strategy in coping with COVID-19: Integrative Review
}

\section{La Enfermera en la Estrategia de Salud de la Familia frente al COVID-19: Revisión Integradora}

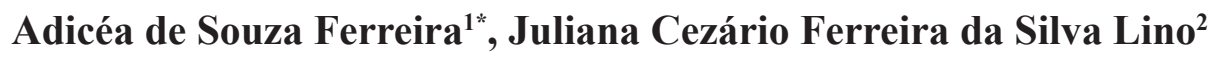

Como citar esse artigo. Ferreira, AS; Lino, JCFS. O Enfermeiro na Estratégia Saúde da Família no enfrentamento da COVID-19: Revisão Integrativa. Revista Pró-UniverSUS. 2020 Jul./Dez.; 11 (2): 65-71.

\begin{abstract}
Resumo
Introdução: Trata-se de um estudo bibliográfico de abordagem qualitativa que tem como objetivo caracterizar o papel do enfermeiro atuante na Estratégia Saúde da Família no enfrentamento da COVID-19. Tendo em vista a probabilidade e rápida propagação da COVID-19, o enfermeiro dentro da Estratégia Saúde da Família contribui de forma relevante para o enfrentamento e combate à doença. Metodologia: os dados foram extraídos da Biblioteca Virtual de Saúde no período de julho a agosto de 2020, em base de dados indexadas. Utilizou-se os descritores: "Infecções por Coronavirus"; "Estratégia Saúde da Família" e "Enfermagem". Foram selecionados, no total, 8 artigos para análise. A descrição do processo se deu através do fluxograma Prisma. Resultados e Discussão: Os artigos foram unânimes quanto a importância da figura do enfermeiro no embate a doença. Diante desse contexto, o enfermeiro na ESF está na vanguarda na prevenção e promoção à saúde nos casos da COVID-19, enfrentando sérias implicações para que suas condições de trabalho e sua segurança pessoal sejam reconhecidas. Entretanto, para além desse reconhecimento, precisam ser traduzidas em políticas eficazes, de suporte e consideração permanente a esses profissionais que estão travando uma luta contra o vírus, colocando-se em risco para a contenção da pandemia. Conclusão: Percebe-se que, no surgimento da COVID-19, faz-se necessário o desenvolvimento de estudos na Estratégia Saúde da Família para esclarecer seus impactos na comunidade e no sistema de saúde. Sabe-se que as recomendações e protocolos serão revistos continuamente, logo, torna-se imprescindível aos pesquisadores, gestores e profissionais da saúde a atualização e revisão constante desses documentos, indispensáveis para a prática do enfermeiro atuante na Estratégia Saúde da Família.

Palavras-chave: Infecções por Coronavirus, Estratégia Saúde da Família, Enfermagem.
\end{abstract}

\begin{abstract}
Introduction: This is a bibliographic study with a qualitative approach that aims to characterize the role of nurses working in the Family Health Strategy in coping with COVID-19. In view of the likelihood and rapid spread of COVID-19, nurses within the Family Health Strategy contribute significantly to coping and fighting the disease. Methodology: data were extracted from the Virtual Health Library from July to August 2020, in an indexed database. The descriptors were used: "Coronavirus infections"; "Family Health Strategy" and "Nursing". In total, 8 articles were selected for analysis. The description of the process took place through the flowchart Prisma. Results and Discussion: The articles were unanimous regarding the importance of the figure of the nurse in fighting the disease. In this context, nurses in the FHS are at the forefront in preventing and promoting health in the cases of COVID-19, facing serious implications for their working conditions and personal safety to be recognized. However, in addition to this recognition, they need to be translated into effective policies, support and permanent consideration for these professionals who are fighting a fight against the virus, putting themselves at risk for the containment of the pandemic. Conclusion: It is noticed that, in the appearance of COVID-19, it is necessary to develop studies in the Family Health Strategy to clarify its impacts on the community and the health system. It is known that the recommendations and protocols will be continuously reviewed, so it is essential for researchers, managers and health professionals to constantly update and review these documents, which are essential for the practice of nurses working in the Family Health Strategy.
\end{abstract}

Keywords: Coronavirus Infections, Family Health Strategy, Nursing. 


\section{Resumen}

Introducción: Se trata de un estudio bibliográfico con abordaje cualitativo que tiene como objetivo caracterizar el papel de los enfermeros que trabajan en la Estrategia de Salud de la Familia en el afrontamiento del COVID-19. En vista de la probabilidad y rápida propagación del COVID-19, las enfermeras dentro de la Estrategia de Salud de la Familia contribuyen significativamente a enfrentar y combatir la enfermedad. Metodología: los datos se extrajeron de la Biblioteca Virtual en Salud de julio a agosto de 2020, en una base de datos indexada. Se utilizaron los descriptores: "Infecciones por coronavirus"; "Estrategia de Salud de la Familia" y "Enfermería”. En total, se seleccionaron 8 artículos para su análisis. La descripción del proceso se realizó a través del diagrama de flujo Prisma. Resultados y Discusión: Los artículos fueron unánimes en cuanto a la importancia de la figura del enfermero en la lucha contra la enfermedad. En este contexto, los enfermeros de la ESF están a la vanguardia en la prevención y promoción de la salud en los casos de COVID-19, enfrentando serias implicaciones para que sus condiciones laborales y seguridad personal sean reconocidas. Sin embargo, además de este reconocimiento, es necesario que se traduzcan en políticas efectivas, de apoyo y consideración permanente a estos profesionales que luchan en la lucha contra el virus, poniéndose en riesgo por la contención de la pandemia. Conclusión: Se advierte que, ante la aparición de COVID-19, es necesario desarrollar estudios en la Estrategia Salud de la Familia para esclarecer sus impactos en la comunidad y el sistema de salud. Se sabe que las recomendaciones y protocolos serán revisados continuamente, por lo que es fundamental que los investigadores, gestores y profesionales de la salud actualicen y revisen constantemente estos documentos, que son fundamentales para la práctica de los enfermeros que trabajan en la Estrategia Salud de la Familia.

Palabras clave: Infecciones por coronavirus, Estrategia de salud familiar, Enfermería.

\section{Introdução}

O Brasil está passando pela mais grave pandemia de uma doença infecciosa causada por um novo coronavírus (SARS-CoV-2). A doença chamada COVID-19 é potencialmente fatal e representa o mais importante problema mundial de saúde pública dos últimos 100 anos, comparado apenas com a gripe espanhola que matou cerca de 25 milhões de pessoas entre 1918 e $1920^{1}$.

A pandemia pelo SARS-CoV-2 teve início na cidade de Wuhan, região central da China no final do ano de 2019, relacionada a transmissão em um mercado de frutos do mar e de animais vivos. Rapidamente se alastrou para toda China, a Ásia e, em dois meses, atingiu todos os continentes ${ }^{1}$.

Em 30 de janeiro de 2020, após reunião com especialistas, a Organização Mundial da Saúde (OMS) declarou Emergência de Saúde Pública de Importância Internacional (ESPII) em razão do espalhamento da COVID-19².

Em 3 de fevereiro de 2020, o Ministério da Saúde do Brasil declarou Emergência de Saúde Pública de Importância Nacional (ESPIN), em decorrência da infecção humana pelo Covid-19, por meio da Portaria MS $n^{\circ} 188 / 2020$. O país, bem como outros estados membros da OMS, está monitorando o surgimento de casos, comportamento da doença e as orientações quanto as medidas para sua minimização e propagação ${ }^{2}$.

A doença é transmitida por meio do contato de gotículas da boca e do nariz que podem ser repassadas por toque ou por meio de objetos e superfícies que estejam contaminados. Em média, o período de incubação da COVID-19 é estimado em 5 a 6 dias, mas pode variar de 0 a 14 dias. Ou seja, o vírus pode levar até 14 dias para manifestar sintomas em uma pessoa que foi infectada ${ }^{3}$.

Normalmente, o quadro clínico da doença apresentado pela pessoa é similar à síndrome gripal, com tosse, febre, dor no corpo, e por vezes dor de cabeça e/ou diarreia. Entretanto, no agravamento do caso, o indivíduo passa a sofrer complicações principalmente pulmonares, ocasionando dificuldade respiratória. A taxa de letalidade é variável de um país para outro. $\mathrm{Na}$ China, por exemplo, em abril de 2020, a taxa era de $3 \%$; no Brasil, no mesmo período, a taxa alcançava $6,3 \%{ }^{4}$.

Com a difusão da COVID-19 para todos os continentes, em um curto período de tempo, esta patologia se transformou em uma pandemia. Em abril de 2020 já havia mais de 2,5 milhões de infectados em todo o mundo; e no Brasil, até o dia 25 de abril, o número já ultrapassava os 50.000 casos. Atualmente, o cenário é de incertezas e desafios; e ainda pouco se sabe sobre a doença e quais suas consequências ao longo prazo ${ }^{4}$.

A Estratégia Saúde da Família (ESF), que nos anos 90 era classificada Programa Saúde da Família (PSF), é considerada uma das principais portas de entrada dos usuários no Sistema Único de Saúde (SUS). A ESF surge como fortalecimento da Atenção Básica (AB) dando acesso à população vulnerável de um território adstrito. Com o surgimento da pandemia da Covid-19 é importante valorizar a atuação do enfermeiro na ESF, visto que esse profissional colabora com as ações de promoção e prevenção à saúde dos sujeitos em âmbito individual e coletivo.

Posto isso, o enfermeiro como integrante da equipe na ESF tem como atribuição: orientar profissionais e usuários quanto controle e agravos que a COVID-19 pode causar, fazer a notificação, monitorar e identificar os casos suspeitos. Sendo assim, esse estudo tem como objetivo: caracterizar o papel do enfermeiro atuante na Estratégia Saúde da Família frente à pandemia da COVID-19.

\section{Metodologia}

Trata-se de um estudo bibliográfico qualitativo e descritivo e os dados foram extraídos da Biblioteca 
Virtual de Saúde (BVS) no período julho e agosto de 2020, em base de dados indexadas. Utilizou-se os descritores: "Infecções por Coronavirus"; "Estratégia Saúde da Família" e "Enfermagem".

O cruzamento dos pares foi executado em duas amostras: a primeira amostra cruzou os descritores: "Infecções por Coronavirus" AND "Enfermagem", obtendo 503 artigos inicialmente. Após a aplicação do teste de relevância 1, em que foram excluídos os artigos que não estavam em português, inglês e espanhol, textos que não estavam disponíveis e trabalhos publicados antes de 2015, restaram 412 artigos. Após a aplicação do teste de relevância 2 , em que foram excluídos temas descontextualizados e repetidos, sobraram 05 artigos.

A segunda amostra cruzou os descritores "Infecções por Coronavirus" AND "Estratégia Saúde da Família" obtendo 19 artigos inicialmente. Após a aplicação do teste de relevância 1 , em que foram excluídos os artigos que não estavam em português, inglês e espanhol, textos que não estavam disponíveis e trabalhos publicados antes de 2015, restaram 16 artigos. Após a aplicação do teste de relevância 2 , em que foram excluídos temas descontextualizados e repetidos, sobraram 03 artigos.

Utilizou-se como critério de inclusão: artigos científicos disponíveis em textos completos; em português, inglês e espanhol; recorte temporal do ano de 2015 a 2020. Como critério de exclusão: textos incompletos e com recorte temporal inferior a 2015. Além dos 08 estudos apresentados no quadro 1 e 2, acrescentamos mais 08 referências que responderam a proposta e o objetivo do estudo, utilizados no presente estudo o total de 16 referências bibliográficas.

Elaborou-se o relatório da revisão a partir das recomendações do Prisma Statment, para a organização e relevância do estudo 5 .

Figura 1. Fluxograma PRISMA como etapas de busca da revisão integrativa com o cruzamento dos pares primeira amostra.
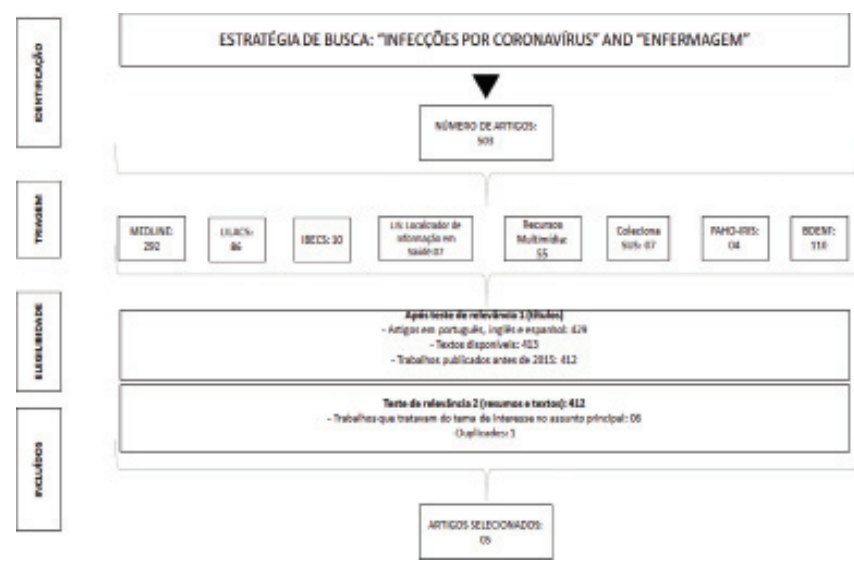

Fonte: Elaboração própria, 2020

\section{Resultados}

Através do fluxograma PRISMA construído nas figuras 1 e 2 e no quadro 1 , seguem as buscas e os artigos utilizados no estudo conforme descritos na metodologia.

\section{Discussão}

A rápida dispersão da COVID-19 tornou-se preocupante para a Estratégia Saúde da Família e para os diversos equipamentos de serviços à saúde. Assim, propõe-se que as estratégias de enfrentamento abranjam aos usuários e os profissionais. O enfermeiro contribui de forma significativa com ações de saúde ${ }^{6}$.

$O$ papel do enfermeiro na ESF tem como finalidade a organização do processo de trabalho para a redução da contaminação da patologia no território, visto que esse profissional atua nas relações com os sujeitos na comunidade e unidade de saúde.

Com a evolução da COVID-19 em um curto espaço de tempo, o seu enfrentamento se tornou prioridade por se tratar de uma emergência sanitária no Brasil e no mundo. Na ESF, o enfermeiro trabalha no incremento quanto às medidas de controle da COVID19, com identificação precoce dos sinais e sintomas e realizando manejo adequado.

No espaço da ESF o enfermeiro possui várias atribuições, entre elas assistência integral às pessoas e famílias, realiza consulta de enfermagem, solicitação de exames complementares, prescrição de medicamentos e atividades educativas; e tem seu dever essencial numa pandemia, desempenhando ações para proteger a saúde das pessoas e salvar vidas?

Entre as atribuições, destaca-se: a consulta de enfermagem é uma atividade privativa do enfermeiro, foi legalizada em junho de 1986, com a lei número 7.498, do exercício profissional de Enfermagem e pela

Figura 2. : Fluxograma PRISMA como etapas de busca da revisão integrativa com o cruzamento dos pares da segunda amostra.

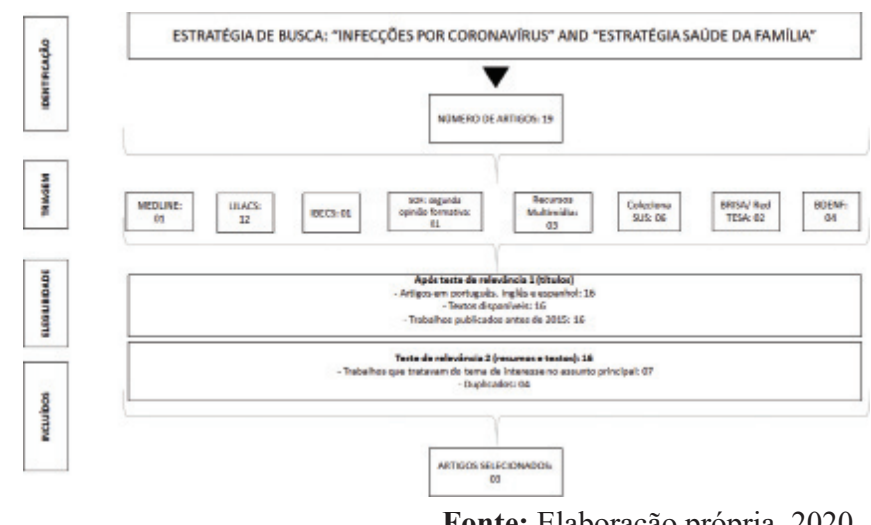

Fonte: Elaboração própria, 2020. 
Quadro 1. Caracterização dos estudos através do fluxograma PRISMA.

\begin{tabular}{|c|c|c|c|c|c|}
\hline ESTUDOS & TÍTULO & ANO & OBJETIVOS & RESULTADOS & CONCLUSÃO \\
\hline E1 & $\begin{array}{c}\text { Desafios da enfer- } \\
\text { magem brasileira no } \\
\text { combate da COVID- } \\
19 .{ }^{8}\end{array}$ & 2020 & $\begin{array}{l}\text { Refletir sobre desa- } \\
\text { fios enfrentados pela } \\
\text { Enfermagem brasi- } \\
\text { leira no combate à } \\
\text { COVID- } 19 .\end{array}$ & $\begin{array}{l}\text { A categoria profissional en- } \\
\text { contra-se na linha de frente no } \\
\text { combate a pandemia, com alto } \\
\text { risco de exposição ao vírus. Os } \\
\text { trabalhadores, maioria sexo fe- } \\
\text { minino, estão trabalhando com } \\
\text { medo, sob pressão, adoecendo e } \\
\text { muitos morrendo. }\end{array}$ & $\begin{array}{l}\text { O cenário pandêmico acen- } \\
\text { tuou os mais diversos riscos } \\
\text { e problemas enfrentados dia- } \\
\text { riamente pelos trabalhadores, } \\
\text { entretanto, o compromisso } \\
\text { com o cuidado biopsicossocial } \\
\text { dos pacientes, família e comu- } \\
\text { nidade sempre se mantém in- } \\
\text { dependentemente da situação } \\
\text { vivenciada. }\end{array}$ \\
\hline E2 & $\begin{array}{l}\text { Enfermagem em con- } \\
\text { texto de pandemia no } \\
\text { Brasil: docilidade dos } \\
\text { corpos em questão. }^{14}\end{array}$ & 2020 & $\begin{array}{c}\text { Discutir o lugar da } \\
\text { categoria profissio- } \\
\text { nal de Enfermagem } \\
\text { no enfrentamento à } \\
\text { pandemia pelo novo } \\
\text { Coronavírus no Bra- } \\
\text { sil, questionando a } \\
\text { docilidade dos cor- } \\
\text { pos. }\end{array}$ & $\begin{array}{c}\text { A profissão de Enfermagem é } \\
\text { essencial em todos os níveis de } \\
\text { atenção à saúde e suas práticas } \\
\text { são indispensáveis em contex- } \\
\text { tos de pandemia, mas tem sido } \\
\text { permeada pela docilidade dos } \\
\text { corpos que, por força dos me- } \\
\text { canismos disciplinadores, tem } \\
\text { sido colocada à extrema vulne- } \\
\text { rabilização. }\end{array}$ & $\begin{array}{l}\text { A politização e o engajamen- } \\
\text { to da categoria são estratégias } \\
\text { potenciais contra os sistemas } \\
\text { de docilidade dos corpos, ca- } \\
\text { paz de tornar a profissão alvo } \\
\text { do respeito e da referência por } \\
\text { parte de líderes políticos, ges- } \\
\text { tores e da sociedade em geral, } \\
\text { quer seja no contexto da pan- } \\
\text { demia ou no cotidiano da vida } \\
\text { humana, pois onde há vida, há } \\
\text { cuidado e há Enfermagem. }\end{array}$ \\
\hline E3 & $\begin{array}{l}\text { Enfermagem em tem- } \\
\text { pos da COVID-19 no } \\
\text { Brasil: um olhar da } \\
\text { gestão do trabalho. }^{15}\end{array}$ & 2020 & $\begin{array}{l}\text { Analisar a situação } \\
\text { da equipe de Enfer- } \\
\text { magem no contexto } \\
\text { da pandemia no Bra- } \\
\text { sil, tendo como foco } \\
\text { a gestão do trabalho } \\
\text { desses profissionais. }\end{array}$ & $\begin{array}{l}\text { Há inúmeras fragilidades nas } \\
\text { condições de emprego, renda, } \\
\text { trabalho, saúde física e mental } \\
\text { desses profissionais. Em rela- } \\
\text { ção à pandemia da COVID-19, } \\
\text { o quadro é de doença instalada } \\
\text { em todo o país, mas diferindo } \\
\text { significativamente entre estados } \\
\text { e regiões. O país já contabiliza } \\
5.533 \text { casos confirmados e } 138 \\
\text { óbitos entre profissionais da } \\
\text { equipe. }\end{array}$ & $\begin{array}{l}\text { A pandemia reforçou com } \\
\text { maior amplitude as precárias } \\
\text { condições de trabalho dos pro- } \\
\text { fissionais da Enfermagem bra- } \\
\text { sileira, sendo necessários mais } \\
\text { estudos e diagnósticos, sobre } \\
\text { os efeitos que incidem no pro- } \\
\text { cesso de trabalho durante o pe- } \\
\text { ríodo atual e no pós-pandemia } \\
\text { para o melhor entendimento da } \\
\text { realidade posta e exposta. }\end{array}$ \\
\hline E4 & $\begin{array}{l}\text { Impacto da COVID- } \\
19 \text { sob o trabalho da } \\
\text { enfermagem brasi- } \\
\text { leira: aspectos epide- } \\
\text { miológicos. }{ }^{16}\end{array}$ & 2020 & $\begin{array}{l}\text { Analisar aspectos } \\
\text { epidemiológicos da } \\
\text { infecção por COVID- } \\
19 \text { nos profissionais } \\
\text { de Enfermagem du- } \\
\text { rante a emergência } \\
\text { da pandemia no ter- } \\
\text { ritório brasileiro em } \\
2020\end{array}$ & $\begin{array}{l}\text { Houve } 8.399 \text { suspeitos, sendo } \\
1.750 \text { confirmados laborato- } \\
\text { rialmente. A maioria dos pro- } \\
\text { fissionais são jovens, do sexo } \\
\text { feminino, residentes em todos } \\
\text { os Estados, com maior con- } \\
\text { centração em São Paulo, Rio } \\
\text { de Janeiro, Santa Catarina, Ce- } \\
\text { ará, Rio Grande do Sul, Minas } \\
\text { Gerais, Pernambuco e Bahia. } \\
\text { A atuação profissional predo- } \\
\text { minou em ambiente hospitalar } \\
\text { e a distribuição temporal dos } \\
\text { casos e óbitos confirmados por } \\
\text { COVID-19 apresentou compor- } \\
\text { tamento exponencial. }\end{array}$ & $\begin{array}{l}\text { A sensibilização das equipes } \\
\text { de Enfermagem quanto a noti- } \\
\text { ficação de irregularidades e de } \\
\text { casos é um importante recurso } \\
\text { para que haja a intensificação } \\
\text { de medidas fiscalizatórias e } \\
\text { adesão efetiva das medidas } \\
\text { preventivas preconizadas, e } \\
\text { consequentemente haverá pre- } \\
\text { servação de vidas dos profis- } \\
\text { sionais de Enfermagem e co- } \\
\text { munidades sob seus cuidados. }\end{array}$ \\
\hline
\end{tabular}


Quadro 1 (cont.). Caracterização dos estudos através do fluxograma PRISMA.

\begin{tabular}{|c|c|c|c|c|c|}
\hline ESTUDOS & TÍTULO & ANO & OBJETIVOS & RESULTADOS & CONCLUSÃO \\
\hline E5 & $\begin{array}{l}\text { Diagnósticos de en- } \\
\text { fermagem para pa- } \\
\text { cientes com COVID- } \\
19 .{ }^{4}\end{array}$ & 2020 & $\begin{array}{c}\text { Elencar com base nas } \\
\text { manifestações clí- } \\
\text { nicas da doença, os } \\
\text { principais diagnósti- } \\
\text { cos de enfermagem } \\
\text { que podem ser apli- } \\
\text { cados para crianças, } \\
\text { adultos, gestantes e } \\
\text { idosos com COVID- } \\
19 .\end{array}$ & $\begin{array}{l}\text { Conforme os sinais e sintomas } \\
\text { presentes na fase aguda da do- } \\
\text { ença em pacientes sob inter- } \\
\text { nação e em uso de terapia me- } \\
\text { dicamentosa, os diagnósticos } \\
\text { predominantes foram proteção } \\
\text { ineficaz relacionado à incapaci- } \\
\text { dade de proteção contra agente } \\
\text { infeccioso evidenciado por tos- } \\
\text { se, calafrios e fadiga, presença } \\
\text { de tosse, Hipertermia relacio- } \\
\text { nada a processo infeccioso } \\
\text { evidenciado por pele quente ao } \\
\text { toque, padrão respiratório inefi- } \\
\text { caz relacionado à dor e fadiga } \\
\text { evidenciado por dispneia. }\end{array}$ & $\begin{array}{l}\text { Alguns diagnósticos são pre- } \\
\text { valentes, entretanto, a avalia- } \\
\text { ção deve ser individual, bem } \\
\text { como a assistência, baseada } \\
\text { nas necessidades de cada in- } \\
\text { divíduo. }\end{array}$ \\
\hline E6 & $\begin{array}{l}\text { Coordenação do cui- } \\
\text { dado, vigilância e } \\
\text { monitoramento de } \\
\text { casos da COVID-19 } \\
\text { na atenção primária à } \\
\quad \text { saúde. } \\
\end{array}$ & 2020 & $\begin{array}{l}\text { Descrever as ações } \\
\text { estratégicas de coor- } \\
\text { denação do cuidado, } \\
\text { monitoramento e e } \\
\text { vigilância dos casos } \\
\text { da COVID-19 na } \\
\text { Atenção Primária à } \\
\text { Saúde. }\end{array}$ & $\begin{array}{c}\text { Papel da Atenção Primária à } \\
\text { Saúde na coordenação do cuida- } \\
\text { do, vigilância e monitoramento } \\
\text { de casos COVID-19 no territó- } \\
\text { rio sanitário, e no ordenamento } \\
\text { da Rede de Atenção à Saúde; a } \\
\text { importância do isolamento so- } \\
\text { cial horizontal e o isolamento } \\
\text { dos casos positivos no próprio } \\
\text { lar, para o controle da COVID- } \\
19 .\end{array}$ & $\begin{array}{l}\text { A Atenção Primária à Saú- } \\
\text { de tem papel estratégico nas } \\
\text { ações de combate à COVID- } \\
19 \text { no território, sobretudo na } \\
\text { redução da transmissão comu- } \\
\text { nitária, na resposta às deman- } \\
\text { das e monitoramento dos casos } \\
\text { e na vigilância em cada fase da } \\
\text { pandemia. }\end{array}$ \\
\hline E7 & $\begin{array}{l}\text { Agentes comunitá- } \\
\text { rios de saúde frente } \\
\text { à COVID-19: vivên- } \\
\text { cias junto aos profis- } \\
\text { sionais de enferma- } \\
\text { gem. }{ }^{13}\end{array}$ & 2020 & $\begin{array}{l}\text { Descrever a experi- } \\
\text { ência vivenciada por } \\
\text { enfermeiras em co- } \\
\text { nexão com Agentes } \\
\text { comunitários de saú- } \\
\text { de para enfrentamen- } \\
\text { to local da pandemia } \\
\text { COVID-19. }\end{array}$ & $\begin{array}{l}\text { Esses profissionais têm sido } \\
\text { protagonistas importantes nas } \\
\text { atividades realizadas dentro } \\
\text { das Estratégias de Saúde da } \\
\text { Família, fortalecendo assim o } \\
\text { trabalho da equipe bem como o } \\
\text { controle e combate da infecção } \\
\text { pelo novo Coronavírus. }\end{array}$ & $\begin{array}{l}\text { Contar com o trabalho desses } \\
\text { profissionais nos territórios } \\
\text { tem sido estratégico e repre- } \\
\text { senta um diferencial para o } \\
\text { cuidado, por auxiliarem no } \\
\text { controle da disseminação e no } \\
\text { monitoramento de grupos de } \\
\text { risco, bem como das pessoas } \\
\text { infectadas pela doença. }\end{array}$ \\
\hline E8 & $\begin{array}{l}\text { Estágio curricular } \\
\text { supervisionado em } \\
\text { enfermagem durante } \\
\text { a pandemia de Coro- } \\
\text { navírus: experiências } \\
\text { na atenção básica. }{ }^{11}\end{array}$ & 2020 & $\begin{array}{l}\text { Relatar as experiên- } \\
\text { cias de estudantes de } \\
\text { enfermagem durante } \\
\text { o estágio curricular } \\
\text { supervisionado na } \\
\text { atenção básica no ce- } \\
\text { nário da pandemia de } \\
\text { Coronavírus. }\end{array}$ & $\begin{array}{l}\text { As atividades desenvolvidas } \\
\text { pelas estudantes mostraram os } \\
\text { desafios que envolvem a profis- } \\
\text { são decorrentes da mudança da } \\
\text { rotina, alterações no processo } \\
\text { de trabalho, de novos protoco- } \\
\text { los e a carência de insumos. No } \\
\text { entanto, contribuiu na forma- } \\
\text { ção da identidade profissional } \\
\text { e possibilitou às estudantes a } \\
\text { oportunidade de vivenciar ex- } \\
\text { periências no contexto }\end{array}$ & $\begin{array}{l}\text { O estágio curricular supervi- } \\
\text { sionado favoreceu o cresci- } \\
\text { mento pessoal, no resgate da } \\
\text { autonomia, no exercício da } \\
\text { liderança e na tomada de de- } \\
\text { cisão. }\end{array}$ \\
\hline
\end{tabular}

Fonte: Elaboração própria, 2020.

\section{Resolução COFEN 159/93 que dispõe sobre a Consulta} de Enfermagem ${ }^{7}$.

Diante desse contexto, o enfermeiro na ESF está na vanguarda na prevenção e promoção à saúde nos casos da COVID-19, enfrentando sérias implicações para que suas condições de trabalho e sua segurança pessoal sejam reconhecidas. Entretanto, para além desse reconhecimento, precisam ser traduzidas em políticas eficazes, de suporte e consideração permanente a esses profissionais que estão travando uma luta contra o vírus, colocando-se em risco para a contenção da pandemia ${ }^{8}$.

Sendo assim, a atuação do enfermeiro na ESF corrobora com os cuidados no enfrentamento da COVID-19 junto á equipe de saúde a qual está inserida, desenvolvendo sua representividade quanto as ações em saúde.

Em decorrência da falta de medicamentos ou vacinas específicas, faz-se necessária a utilização de 
medidas não-farmacológicas para controlar o contágio da COVID-19, tendo como exemplo a higienização das mãos, por meio da lavagem com água e sabão ou antissepsia utilizando gel à base de álcool $70 \%{ }^{9}$.

Faz-se importante também seguir as três medidas clássicas de saúde pública, já utilizadas há muito tempo no controle de epidemias, mas que ainda geram dúvidas na população. São elas: o isolamento de contaminados e contatos domiciliares por 14 dias, a quarentena, com a restrição do movimento de pessoas expostas a uma doença contagiosa não infectadas ou em estágio de incubação e o distanciamento social, por meio da redução das interações entre pessoas de uma comunidade, em decorrência da possibilidade de contaminação, pois muitos indivíduos podem estar infectados, mesmo que não diagnosticados. Essas medidas visam a reduzir a propagação da doença ${ }^{9}$.

A epidemia da COVID-19 exige a invenção criativa de novos modos de cuidados que devem estar aliados a ESF e dos princípios doutrinários e organizativos do Sistema Único de Saúde (SUS), sendo o enfermeiro articulador no enfrentamento da COVID19 na ESF.

Para tal situação, é fundamental o uso de equipamento de proteção individual (EPI) como máscara, luvas, protetor ocular ou face shield e avental descartável de mangas longas. Esses EPIs auxiliam na proteção dos profissionais ao contato com o usuário.

O manejo clínico do caso suspeito de COVID-19 na ESF envolve a identificação imediata dos usuários com sintomas respiratórios, a adoção de medidas para evitar contágio, a estratificação da gravidade da síndrome gripal, o atendimento dos casos leves, a prescrição e a orientação quanto ao isolamento domiciliar, a estabilização e o encaminhamento dos casos graves a serviços de urgência, se necessário. O enfermeiro nesse sentido deve realizar essas intervenções para enfrentar a COVID-19, dando resolutividade aos casos ${ }^{10}$.

Com relação a atuação do enfermeiro no ESF, o acolhimento e o vínculo facilitam as orientações de saúde com foco na prevenção de COVID-19 e na promoção da saúde e precisam contemplar ações de promoção da saúde, prevenção de doenças, tratamento e a reabilitação ${ }^{11}$.

Para atender as demandas emanadas com à COVID-19, necessitamos de uma ESF que recupere seus princípios de base comunitária, trabalho em equipe, intra e extramuros, com usuários, pacientes, famílias e comunidade, visando a integralidade e a humanidade das ações em toda linha de cuidado para o enfretamento da COVID-19 que se configura um desafio da atual realidade da Estratégia Saúde da Família.

\section{Considerações Finais}

$\mathrm{O}$ enfermeiro em exercício na ESF diante do enfrentamento da COVID-19, torna-se elemento contributivo no processo de trabalho do desenvolvimento de suas ações de não propagação da doença adentro da ESF, ao realizar seu papel participante no combate à doença.

Destaca-se nesse trabalho a valorização do profissional enfermeiro no cuidado em saúde, principalmente diante da pandemia. Através de estudos e pesquisas, a profissão se fortalece ainda mais, buscando mais reconhecimento e direitos dentro de sua atuação.

Como a COVID-19 surgiu recentemente, faz-se necessário o desenvolvimento de estudos na ESF para esclarecer seus impactos na comunidade e no sistema de saúde. Sabe-se que as recomendações e protocolos serão revistos continuamente, logo, torna-se imprescindível aos pesquisadores, gestores e profissionais da saúde a atualização e revisão constante desses documentos, indispensáveis para a prática.

Salienta-se que, com esse estudo de revisão integrativa, pode-se compartilhar os achados das literaturas em destaque ao proporcionar a interseção do enfermeiro na ESF na prevenção e promoção em saúde do combate da COVID-19.

\section{Referências}

1. Rothan HA, Byrareddy SN. The epidemiology and pathogenesis of coronavirus disease (COVID-19) outbreak. J Autoimmun. 2020;109:102433. https://pubmed.ncbi.nlm.nih.gov/32113704/

2. COFEN. Nota Técnica $\mathrm{N}^{\circ} 01 / 2020$ : Orientações sobre o novo Coronavírus (COVID-19) Sistema COFEN/Conselhos Regionais em Foco. 2020.http://biblioteca.cofen.gov.br/wp-content/uploads/2020/06/Notat\%C3\%A9cnica-n \%C2\%BA-012020.-Orienta\%C3\%A7\%C3\%B5es-sobreo-novocoronav $\% \mathrm{C} 3 \%$ ADrus-Covid-19.pdf

3. Duarte RB et al,. Agentes Comunitários de Saúde frente à COVID19: Vivências junto aos profissionais de enfermagem. Enferm. Foco 2020; 11 (1) Especial: 252-256.http://revista.cofen.gov.br/index.php/enfermagem/ article/view/3597

4. Dantas TP, Aguiar CAS, Rodrigues VRT, Silva RRG, Silva MIC, Sampaio LRL, et al. Diagnósticos de enfermagem para pacientes com COVID-19 Journal Health NPEPS. 2020 jan-jun; 5(1):396-416.https:// periodicos.unemat.br/index.php/jhnpeps/article/download/4575/3617

5. Moher D, Liberati A, Tetzlaff J, Altman DG, ThePrisma Group. Preferred Reporting Items for Systematic Reviews and Meta-Analyses: The Prisma Statement. Epidemiol. Serv. Saúde. 2015; 24(2):335-42.https://www. scielo.br/pdf/ress/v24n2/2237-9622-ress-24-02-00335.pdf

6. Cabral ERM, Melo MC, Cesar ID, Oliveira REM, Bastos TF, Machado LO, et al. Contribuições e desafios da Atenção Primária à Saúde frente à pandemia de COVID-19. InterAm J Med Health 2020;3:e202003012. https:// www.iajmh.com/iajmh/article/download/133/163/

7. Cavalcante CCFS, Sousa JAS, Dias AMA. Consulta de Enfermagem aos casos suspeitos de COVID -19, na Atenção Primária a Saúde. Revista da FAESF, vol. 4. Número especial COVID 19. Junho (2020) 34-40.http:// faesfpi.com.br/revista/index.php/faesf/article/view/112/98

8. Oliveira AC. Desafios da enfermagem frente ao enfrentamento da pandemia da Covid19. REME - Rev Min Enferm. 2020. https:/www.reme. org.br/artigo/detalhes/1448

9. Sousa NAR, Freitas DRJ . Utilização de máscaras: Indicações de uso e manejo durante a pandemia da Covid-19. Cogitare enferm. https://revistas. ufpr.br/cogitare/article/view/72867 
10. Fernandez MV, Castro DM, Fernandes LMM, Alves IC. Reorganizar para avançar: a experiência da Atenção Primária à Saúde de Nova Lima/ MG no enfrentamento da pandemia da COVID-19. APS em Revista Vol. 2, n. 2, p. 114-121 | Junho - 2020. https://apsemrevista.org/aps/article/ download/84/60/

11. Souza LB, Schir DG, Soccol KLS, Santos NO, Marchiori MRCT. Estágio curricular supervisionado em enfermagem durante a pandemia de Coronavírus: experiências na atenção básica. J. nurs. health. 2020;10(n. esp.):e20104017.https://periodicos.ufpel.edu.br/ojs2/index.php/ enfermagem/article/view/19050/11638

12. NetoFRGXetal.. Coordenação do cuidado, vigilância emonitoramento de casos da COVID-19 na Atenção Primária à Saúde. Enferm. Foco 2020; 11 (1) Especial: 239-245 http://revista.cofen.gov.br/index.php/enfermagem/ article/view/3682/835

13. Quadros A, Fernandes MTC, Araujo BR, Caregnato RCA. Desafios da enfermagem Brasileira no combate da COVID-19: uma reflexão. Enferm. Foco 2020; 11 (1) Especial: 78-83.http://revista.cofen.gov.br/index.php/ enfermagem/article/view/3748

14. Sousa AR, Olimpio A, Cunha CLF . Enfermagem em contexto de pandemia no Brasil: docilidades dos corpos em questão. Enferm. Foco 2020; 11 (1) Especial: 95-100 http://revista.cofen.gov.br/index.php/enfermagem/ article/view/3499

15. Machado MH,Pereira EJ, Neto FRGX, Mesquita MC, Wermelinger W. Enfermagem em tempos de COVID-19 no Brasil: um olhar da gestão do trabalho. Enferm. Foco 2020; 11 (1). Especial: 32-39. http://revista.cofen. gov.br/index.php/enfermagem/article/view/3994

16. Nascimento VF, Espinosa MM, Silva MCN, Freire NP, Trettel ACPT. Impacto da COVID-19 sob o Trabalho da Enfermagem Brasileira: Aspectos Epidemiológicos. Enferm. Foco 2020; 11 (1). Especial: 24-31. http://biblioteca.cofen.gov.br/wp-content/uploads/2020/08/ImpactoCOVID19Enfermagem.pdf 